\title{
DA PROTEÇÃO DO ÁCIDO ASCÓRBICO "IN VITRO" PELA XANTINA, SUBSTANCIAS CORRELATAS E EXTRATO HEPATICO TOTAL OU NÃO, CONTRA AGENTES DE OXIDAÇÃO
}

\section{Fonseca Ribeiro e Virgilio Bonoldi}

Em recente publicação (1941), GIRI e KRISHNAmURThy (1) estabelecem que a oxidação do ácido ascórbico pelo ion cobre e pelo ar atmosférico é completamente inhibida a $30^{\circ} \mathrm{C}$. e em $\mathrm{pH} 7,2$ quando presentes pequenas quantidades de xantina ou ácido úrico; outras purinas experimentadas do mesmo ponto de vista negaram efeito protetor, concluindo os autores pela especificidade dos derivados da purina que apresentam livre de substituições o imino grupo da posição 7. Trabalhando de preferência com o manometro de Warburg apresentam os seguintes resultados:

Äcido Ascórbico: 2 mgr. Ion cuprico 0,71 gamas.

Volume total: $3 \mathrm{~cm} .^{3}$

\begin{tabular}{|c|c|c|c|c|}
\hline & \multirow{2}{*}{ Substancias } & \multirow{2}{*}{$\begin{array}{l}\text { Concentra- } \\
\text { ção Molar }\end{array}$} & \multicolumn{2}{|c|}{ Oxigenio desprendido } \\
\hline & & & $\begin{array}{c}\text { Tempo en } \\
15\end{array}$ & $\begin{array}{c}\text { minutos } \\
30\end{array}$ \\
\hline $\begin{array}{l}\text { Vitamina } \mathrm{C} \text { e } \\
\text { ion cuprico }\end{array}$ & - & - & 66 & 130 \\
\hline $\begin{array}{l}\text { Vitamina } \mathrm{C} \text { e } \\
\text { ion cuprico }\end{array}$ & Xantina & 0,00017 & 0 & 0 \\
\hline $\begin{array}{l}\text { Vitamina } \mathrm{C} e \\
\text { ion cuprico }\end{array}$ & Ácido úrico & 0,00015 & 0 & 0 \\
\hline $\begin{array}{l}\text { Vitamina C e } \\
\text { ion cuprico }\end{array}$ & Teofilina & 0,00014 & 13 & 17 \\
\hline $\begin{array}{l}\text { Vitamina } \mathrm{C} \text { e } \\
\text { ion cuprico }\end{array}$ & Teobromina & 0,0007 & 65 & 120 \\
\hline $\begin{array}{l}\text { Vitamina C e } \\
\text { ion cuprico }\end{array}$ & Cafeina & 0,00064 & 62 & 121 \\
\hline $\begin{array}{l}\text { Vitamina } \mathrm{C} \text { e } \\
\text { ion cuprico }\end{array}$ & Creatinina & 0,0011 & 5 & 10 \\
\hline $\begin{array}{l}\text { Vitamina } \mathrm{C} \text { e } \\
\text { ion cuprico }\end{array}$ & Creatina & 0,00095 & 62 & 130 \\
\hline
\end{tabular}

Temperatura $=30^{\circ} \mathrm{C}$. 
Já anteriormente foram divulgadas provas da proteção especifica do ácido ascórbico pelo ácido metafosfórico em presença do ion cobre como se vê dos trabalhos de Musulin e King (2), Lyman, Schultz e King (3) confirmados entre nós por O. F. Ribeiro (4).

É fora de duvida que tal estudo apresenta alem do interesse puramente científico, finalidade de aplicação pratica de grande monta, qual seja o da conservação de soluçôes destinadas ao uso terapeutico; e dentre os problemas que se nos afiguram dignos de tentativas de esclarecimentos podemos enumerar:

$\left.1 .^{\circ}\right)$ Pesquisa de outras substancias que por suas caracteristicas fisicas e quimicas pudessem ser de mais vantajoso emprego: pode-se referir que tanto a xantina como o ácido úrico são de muito baixa solubilidade alem de que a primeira destas substancias frequentemente, pelas alterações da temperatura ambiente, se insolubilisa de suas soluções sendo a solução de 70 gamas por $\mathrm{cm} \cdot{ }^{3}$ praticamente saturada;

$2 .^{\circ}$ ) Verificação de que o poder protetor da xantina ou substancia correlata se extende tambem à outros agentes oxidativos do ácido ascórbico, tais como o ion férrico e o peroxido de hidrogenio: embora se podesse aprioristicamente, prever essa generalisação, importa obter, do fato, uma demonstração experimental;

$3 .^{\circ}$ ) Estudo do comportamento das substâncias impedientes da oxidação, face o fermento ascorbinase capaz de promover a formação do ácido dehidroascórbico a partir do ascórbico: seria isso tentar um esclarecimento para o fato observado por Pimenta (4) de que, nas dosagens de vitamina $\mathrm{C}$, diferentes substratos mostram-se com a característica de exigir maior quantidade desse enzima que a calculada para completa oxidação do ácido ascórbico.

Estes foram, primordialmente, os pontos que visamos da feitura de nosso trabalho.

\section{PARTE EXPERIMENTAL}

Como método de determinação do ácido ascórbico utilisamos a titulagem simples pelo 2,6-diclorofenolindofenol, já que trabalhamos sempre com soluções da substância pura; na parte referente ao emprego da ascorbinase utilisamos a técnica recentemente descrita por RIbeiro, Bonoldi e Ribeiro (6), substituindo a modificação de Leser (7) ao processo de TAUbER e KLEINER (8).

Veremos a seguir os nossos resultados:

Trabalhando com xantina por nós obtida a partir do Guano do Perú, registramos os resultados que seguem: 


\section{INFLUENCIA DA XANTINA NA PROTEÇÃO Ȧ OXIDAÇÃO DO ÁCIDO ASCóRBICO}

Temperatura $40^{\circ} \mathrm{C}$.

Solução de sulfato de cobre: um $\mathrm{cm} \cdot{ }^{3}$ contendo um gama de ion cuprico.

Solução de xantina: contendo por $\mathrm{cm} \cdot{ }^{3} 70$ gamas da dioxipurina.

Solução aceto-acetica tampão $0,2 \mathrm{~N}$ : $\mathrm{pH}$ 6: segundo Walpole.

Solução de ácido ascórbico: um $\mathrm{cm} .{ }^{3}$ da qual reduziu $21,5 \mathrm{~cm} .^{3}$ do reativo de Tilmans, F : $0.105: 2.257 \mathrm{mgrs}$. do ácido por $\mathrm{cm}^{3}$

Volume total completado com agua a $10 \mathrm{~cm} \cdot{ }^{3}$, em todos os tubos.

\begin{tabular}{|c|c|c|c|c|c|c|c|c|c|c|}
\hline \multirow{2}{*}{ Nos. } & \multicolumn{4}{|c|}{ Cm. ${ }^{3}$ da solução de } & \multicolumn{3}{|c|}{$\begin{array}{l}\text { Quantidade em mgr. de } \\
\text { ácido ascórbico existente } \\
\text { em } 10 \mathrm{~cm} 3 \text {. depois de }\end{array}$} & \multicolumn{3}{|c|}{$\begin{array}{l}\text { Perdas em porcentagens } \\
\text { depois de }\end{array}$} \\
\hline & $\begin{array}{l}\text { Ácido } \\
\text { ascór. }\end{array}$ & Tampão & $\begin{array}{l}\text { Sulfato } \\
\text { decobre }\end{array}$ & Xantina & & $\begin{array}{l}\text { m3. de } \\
30^{\prime}\end{array}$ & $\begin{array}{r}\text { de } \\
45 \\
\end{array}$ & $15^{\prime}$ & $30^{\circ}$ & 45 \\
\hline 1 & 1 & 1 & - & - & 1,90 & 1,76 & 1,65 & 16 & 22 & 27 \\
\hline 2 & $"$ & " & 1 & - & 1,37 & 1,29 & 1,19 & 39 & 43 & 47 \\
\hline 3 & " & " & - & 2,5 & 2,22 & 2,22 & 2,22 & 1 & 1 & 1 \\
\hline 4 & $"$ & $"$ & 1 & 2,5 & 2,19 & 2,12 & 2,05 & 3 & 6 & 9 \\
\hline 5 & $"$ & " & 1 & 5 & 2,20 & 2,17 & 2,15 & 2 & 4 & 5 \\
\hline 6 & " & " & 3 & 2,5 & 2,13 & 2,06 & 2,02 & 6 & 8 & 10 \\
\hline 7 & " & " & 1 & 7 & 2,19 & 2,14 & 2,10 & 3 & 5 & 7 \\
\hline 8 & " & " & 5 & 2,5 & 2,15 & 2,10 & 2,03 & 5 & 7 & 10 \\
\hline
\end{tabular}

Note-se aquí o extraordinario poder protetor da xantina contra a oxidação do ácido ascórbico; mesmo no tubo 8 em que foram empregados 5 gamas de ion cuprico contra 175 gamas de xantina as perdas foram 0,10 mgr., 0,15 mgr., 0,22 mgr. de ácido ascórbico nos tempos de 15,30 e 45 minutos, o que significa proteção de 95,93 e $90 \%$ respectivamente sôbre o total do ácido ascórbico.

Repetimos agora a prova em presença da ascorbinase.

\section{INFLUENCIA DA XANTINA E ASCORBINASE NA PROTEÇÃO Ã

$$
\text { OXIDAÇÃO DO ÁCIDO ASCORBICO }
$$

Temperatura: $40^{\circ} \mathrm{C}$.

Solução de sulfato de cobre: um $\mathrm{cm}^{3}$ contendo um gama de ion cuprico.

Solução de xantina: contendo por $\mathrm{cm}^{3} 70$ gamas da dioxipurina.

Solução aceto-acetica, tampão, $\mathrm{pH}$ 6: segundo Walpole.

Solução de ácido ascórbico: um cm..$^{3}$ da qual reduziu $17,3 \mathrm{~cm} \cdot{ }^{3}$ do reativo de Tilmans F: 0.105: $1.816 \mathrm{mgr}$. do ácido por $\mathrm{cm} .^{3}$

Volume total completado com agua a $10 \mathrm{~cm} \cdot{ }^{3} \mathrm{em}$ todos os tubos.

\begin{tabular}{|c|c|c|c|c|c|c|c|c|c|c|c|}
\hline \multirow{3}{*}{$\mathrm{N}, "$} & \multicolumn{5}{|c|}{$\mathrm{Cm} .3$ da solução de } & \multirow{2}{*}{\multicolumn{3}{|c|}{$\begin{array}{l}\text { Quantidade de ácido } \\
\text { ascórbico em mgrs. } \\
\text { existente em } 10 \mathrm{~cm} \cdot 3 \\
\text { depois de }\end{array}$}} & \multirow{2}{*}{\multicolumn{3}{|c|}{$\begin{array}{l}\text { Perdas em porcenta- } \\
\text { gens depois de }\end{array}$}} \\
\hline & \multirow{2}{*}{$\begin{array}{l}\text { Aeido } \\
\text { ascór. }\end{array}$} & \multirow{2}{*}{$\begin{array}{c}\text { Tam- } \\
\text { pâo }\end{array}$} & \multirow{2}{*}{$\begin{array}{l}\text { Sulfato } \\
\text { de cobre }\end{array}$} & \multirow{2}{*}{$\begin{array}{l}\text { Xan- } \\
\text { tina }\end{array}$} & \multirow{2}{*}{$\begin{array}{l}\text { Ascor- } \\
\text { binase }\end{array}$} & & & & & & \\
\hline & & & & & & $15^{\prime}$ & $30^{\prime}$ & $45^{\prime}$ & $15^{\prime}$ & $30^{\prime}$ & $45^{\prime}$ \\
\hline 1 & 1 & 1 & - & 一 & 一 & 1,43 & 1,19 & 1,09 & 20 & 34 & 40 \\
\hline 2 & " & " & 1 & - & - & 1,10 & 0,95 & 0,80 & 39 & 48 & 56 \\
\hline 3 & " & " & - & 5 & - & 1,81 & 1,81 & 1,80 & 0 & 0 & 0 \\
\hline 4 & " & ” & 1 & 5 & - & 1,76 & 1,70 & 1,66 & 3 & 6 & 8 \\
\hline 5 & ” & $"$ & - & - & 1 & 0,92 & 0,60 & 0,52 & 49 & 66 & 71 \\
\hline 6 & " & " & 1 & - & 1 & 1,14 & 0,99 & 0,85 & 37 & 45 & 83 \\
\hline 7 & $"$ & " & - & 5 & 1 & 1,03 & 0,87 & 0,61 & 43 & 50 & 55 \\
\hline 8 & ” & ", & 1 & 5 & 1 & 0,89 & 0,59 & 0,54 & 51 & 67 & 70 \\
\hline
\end{tabular}


E' fora de dúvida que ha apenas um pequeno efeito da xantina como inhibidor da ação enzimatica. O que se vê nesse quadro e que merece ser ressaltado é que ambos os agentes oxidantes do ácido ascórbico - o ion cuprico e a ascorbinase - (e que é uma proteina do cobre) eficientes em ação isolada, mostram um certo antagonismo: enquanto que o cobre puro destroi $39,48,56$ por cento do ácido ascórbico (tubo 2), a ascorbinase, nas mesmas condições consumiu 49, 66, 71 por cento (tubo 5) e a mistura dos dois agentes (tubo 6) transformou apenas $37,45,53$ por cento do ácido ascórbico.

Outras substancias revelam os seguintes resultados:

\section{INFLUENCIA DO ÃCIDO ÚRICO E ASCORBINASE NA PROTEÇÃO} À OXIDAÇÃO DO ÁCIDO ASCORBICO

Temperatura $40^{\circ} \mathrm{C}$.

Solução de sulfato de cobre: um $\mathrm{cm} \cdot{ }^{3}$ contendo um gama de ion cuprico.

Solução de ácido úrico: contendo por $\mathrm{cm}^{3} 30$ gamas da trioxipurina.

Solução aceto-acetica, tampão, $0.2 \mathrm{~N}$ : pH 6: segundo Walpole.

Solução de ácido ascórbico: um $\mathrm{cm} .^{3}$ com poder redutor sobre o reativo de Tilmans F : 0,105 igual a $17,3 \mathrm{~cm} \cdot{ }^{3}: 1.816 \mathrm{mgr}$. do ácido por $\mathrm{cm} \cdot{ }^{3}$

Volume total completado com agua a $10 \mathrm{~cm} \cdot{ }^{3}$ em todos os tubos.

\begin{tabular}{|c|c|c|c|c|c|c|c|c|c|}
\hline \multirow[t]{2}{*}{ Noos } & \multicolumn{5}{|c|}{ Cm.3 da solução de } & \multicolumn{2}{|c|}{$\begin{array}{l}\text { Quantidade em } \\
\text { mgr. de ácido ascór- } \\
\text { bico existentes em } \\
10 \mathrm{~cm} .3 \text { depois de } \\
\end{array}$} & \multicolumn{2}{|c|}{$\begin{array}{l}\text { Perdas em porcen- } \\
\text { tagens depois de }\end{array}$} \\
\hline & $\begin{array}{l}\text { Áido } \\
\text { ascór. }\end{array}$ & Tampāo & $\begin{array}{l}\text { Sulfato } \\
\text { de cobre }\end{array}$ & $\begin{array}{l}\text { Ácido } \\
\text { úrico }\end{array}$ & $\begin{array}{l}\text { Ascorbi- } \\
\text { nase }\end{array}$ & $15^{\circ}$ & 30 , & $15^{\circ}$ & $30^{\prime}$ \\
\hline 1 & 1 & 1 & - & - & - & 1,66 & 1,50 & 8 & 17 \\
\hline 2 & " & ” & 1 & - & - & 1,01 & 0,97 & 44 & 46 \\
\hline 3 & ” & ” & - & 5 & - & 1,65 & 1,57 & 9 & 13 \\
\hline 4 & " & ” & 1 & 5 & - & 1,11 & 0,89 & 39 & 51 \\
\hline 5 & $"$ & $"$ & - & - & 1 & 1,01 & 0,76 & 44 & 58 \\
\hline 6 & " & " & 1 & - & 1 & 1,23 & 1,03 & 32 & 43 \\
\hline 7 & ," & " & - & 5 & 1 & 1,23 & 0,95 & 32 & 48 \\
\hline 8 & ” & " & 1 & 5 & 1 & 1,22 & 1,00 & 32 & 45 \\
\hline
\end{tabular}

Por essa experiência confirma-se o fato já verificado a proposito da xantina, de que existe um certo antagonismo entre a oxidação pelo cobre e a ascorbinase. Nota-se mais que é assaz pequena a diferença que o ácido úrico pode produzir no efeito da ascorbinase. 


\section{INFLUENCIA DA CREATININA NA PROTEÇÃO À OXIDAÇÃO DO ÁCIDO ASCÓRBICO}

Temperatura $40^{\circ} \mathrm{C}$.

Solução de sulfato de cobre: um $\mathrm{cm} \cdot{ }^{3}$ contendo um gama de ion cuprico.

Solução de creatinina: um $\mathrm{cm} .^{3}$ contendo 124 gamas do guanidino-derivado.

Solução aceto-acetica, tampão $0.2 \mathrm{~N}$ : $\mathrm{pH}$ 6: segundo Walpole.

Solução de ácido ascórbico: um $\mathrm{cm} \cdot{ }^{3}$ da qual reduziu $21,5 \mathrm{~cm} .{ }^{3}$ do reativo de Tilmans F: 0.105: $2.2575 \mathrm{mgr}$. do ácido por $\mathrm{em} .{ }^{3}$

Volume completado com agua a $10 \mathrm{~cm} \cdot{ }^{3} \mathrm{em}$ todos os tubos.

\begin{tabular}{|c|c|c|c|c|c|c|c|c|c|c|}
\hline \multirow{2}{*}{ N.os } & \multicolumn{4}{|c|}{ Cm.3 da solução de } & \multicolumn{3}{|c|}{$\begin{array}{l}\text { Quantidade em mgrs. de } \\
\text { ácido ascórbico existente } \\
\text { em } 10 \mathrm{~cm} .3 \text { depois }\end{array}$} & \multicolumn{3}{|c|}{$\begin{array}{l}\text { Perdas em porcentagens } \\
\text { depois de }\end{array}$} \\
\hline & $\begin{array}{l}\text { Ácido } \\
\text { ascór. }\end{array}$ & Tampão & $\begin{array}{l}\text { Sulfato } \\
\text { de cobre }\end{array}$ & $\begin{array}{c}\text { Creati- } \\
\text { nina }\end{array}$ & $15^{\prime}$ & 30 & $45^{\circ}$ & $15^{\prime}$ & $30^{\prime}$ & $45^{\prime}$ \\
\hline 1 & 1 & 1 & - & - & 1,78 & 1,72 & 1,65 & 21 & 24 & 27 \\
\hline 2 & $"$ & $"$ & 1 & - & 1,42 & 1,18 & 1,15 & 37 & 48 & 49 \\
\hline 3 & $"$ & $"$ & - & 2,5 & 2,23 & 2,10 & 2,05 & 1 & 7 & 9 \\
\hline 4 & $"$ & $"$ & 1 & 2,5 & 1,92 & 1,66 & 1,44 & 15 & 26 & 36 \\
\hline 5 & $"$ & $"$ & 1 & 5 & 2,07 & 1,96 & 1,85 & 8 & 13 & 18 \\
\hline 6 & $"$ & " & 3 & 2,5 & 2,23 & 2,03 & 1,81 & 1 & 10 & 20 \\
\hline 7 & ” & " & 1 & 7 & 2,20 & 2,13 & 2,00 & 2 & 6 & 12 \\
\hline 8 & $"$ & " & 5 & 2,5 & 1,50 & 1,34 & 1,13 & 34 & 40 & 50 \\
\hline
\end{tabular}

\section{INFLUENCIA DO VERONAL DA PROTEÇÃO Ã OXIDAÇÃO} DO ÁCIDO ASCÓRBICO

Temperatura $40^{\circ} \mathrm{C}$

Solução de sulfato de cobre: um $\mathrm{cm}^{3}$ contendo um gama de ion cuprico.

Solução de Veronal: um $\mathrm{cm} \cdot{ }^{3}$ contendo 200 gamas do ácido.

Solução aceto-acetica, tampão $0.2 \mathrm{~N}$ : $\mathrm{pH}$ 6: segundo Walpole.

Solução de ácido ascórbico: um $\mathrm{cm}^{3}$ da qual reduziu $21.5 \mathrm{~cm} .^{3}$ do reativo de Tilmans F : 0.105: 2.2575 mgrs. do ácido por $\mathrm{cm} .{ }^{3}$

Volume total completado com agua a $10 \mathrm{~cm} .^{3} \mathrm{em}$ todos os tubos.

\begin{tabular}{|c|c|c|c|c|c|c|c|c|}
\hline \multirow[t]{2}{*}{ N.os } & \multicolumn{4}{|c|}{$\mathrm{Cm} .3$ da soluçũo de } & \multicolumn{2}{|c|}{$\begin{array}{l}\text { Quantidade em mgr. } \\
\text { de ácido ascórbico } \\
\text { existente em } 10 \mathrm{~cm} .3 \\
\text { depois de }\end{array}$} & \multicolumn{2}{|c|}{$\begin{array}{l}\text { Perdas em } \\
\text { porcentagem } \\
\text { depois de }\end{array}$} \\
\hline & $\begin{array}{l}\text { Ácido } \\
\text { ascór. }\end{array}$ & Tampão & $\begin{array}{l}\text { Sulfato } \\
\text { de cobre }\end{array}$ & Veronal & $15^{\prime}$ & $30^{\prime}$ & $15^{\prime}$ & $30^{\circ}$ \\
\hline 1 & 1 & 1 & 一 & - & 1,93 & 1,76 & 15 & 22 \\
\hline 2 & $"$ & $"$ & 1 & - & 1,37 & 1,23 & 39 & 45 \\
\hline 3 & $"$ & $"$ & 一 & 2,5 & 1,76 & 1,55 & 22 & 31 \\
\hline 4 & $"$ & ” & 1 & 2,5 & 1,45 & 1,26 & 35 & 44 \\
\hline 5 & $"$ & $"$ & 1 & 5 & 1,39 & 1,27 & 38 & 44 \\
\hline 6 & $"$ & $"$ & 3 & 2,5 & 1,36 & 1,23 & 40 & 46 \\
\hline 7 & $"$ & $"$ & 1 & 7 & 1,48 & 1,35 & 35 & 40 \\
\hline 8 & $"$ & $"$ & 5 & 2,5 & 1,34 & 1,18 & 40 & 48 \\
\hline
\end{tabular}


Temperatura: $40^{\circ} \mathrm{C}$.

INFLUENCIA DA GUANINA NA PROTEÇÃO A OXIDAÇÃO DO ÁCIDO ASCÓRBICO

Solução de sulfato de cobre: um $\mathrm{cm}^{3}$ contendo um gama de ion cuprico.

Solução de Guanina: contendo por $\mathrm{cm} \cdot{ }^{3} 70$ gamas.

Solução aceto-acetica, tampão $0.2 \mathrm{~N}: \mathrm{pH}$, segundo Walpole.

Solução de ácido ascórbico: tendo: por $\mathrm{cm} .^{3}$ quantidade tal que reduziu $11.4 \mathrm{~cm} .{ }^{3}$ do reativo de Tilmans F: 0.105: $1.197 \mathrm{mgr}$. de ácido ascórbico por $\mathrm{cm} .{ }^{3}$.

Volume total completado com agua a $10 \mathrm{~cm} \cdot{ }^{3}$ em todos os tubos.

\begin{tabular}{|c|c|c|c|c|c|c|c|c|c|c|c|}
\hline \multirow[t]{2}{*}{ N.os } & \multicolumn{5}{|c|}{ Cm.: da soluçüo de } & \multicolumn{3}{|c|}{$\begin{array}{l}\text { Quantidade em mgrs. de } \\
\text { ácido ascórbico exis- } \\
\text { tente em } 10 \mathrm{~cm} .3 \\
\text { depois de }\end{array}$} & \multicolumn{3}{|c|}{$\begin{array}{l}\text { Perdas em porcenta- } \\
\text { gens depois de }\end{array}$} \\
\hline & $\begin{array}{l}\text { Ácido } \\
\text { aseór. }\end{array}$ & $\begin{array}{c}\text { Tam- } \\
\text { pão }\end{array}$ & $\begin{array}{r}\text { Sulfato } \\
\text { de cobre }\end{array}$ & $\begin{array}{l}\text { Gua- } \\
\text { nina }\end{array}$ & $\begin{array}{l}\text { Ascor- } \\
\text { binase }\end{array}$ & $15^{\prime}$ & $30^{\prime}$ & $45^{\prime}$ & $15^{\circ}$ & $30^{\circ}$ & $45^{\circ}$ \\
\hline 1 & 1 & 1 & 一 & - & - & 0,85 & 0,71 & 0,52 & 29 & 40) & 56 \\
\hline 2 & " & " & 1 & - & - & 0,48 & 0,34 & 0,27 & 6 () & 70 & 77 \\
\hline 3 & $"$ & $"$ & - & 5 & - & 1,10 & 1,08 & 1,05 & 8 & 10) & 12 \\
\hline 4 & " & ", & 1 & 5 & - & 1,10 & 1,05 & 0,99 & 8 & 12 & 17 \\
\hline 5 & " & ” & - & - & 1 & 0,02 & 0,00 & 0,00 & 98 & 100 & 100 \\
\hline 6 & " & " & 1 & - & 1 & 0,22 & 0,08 & 0,00 & 82 & 83 & 100 \\
\hline 7 & $"$ & ” & - & 5 & 1 & 0,30 & 0,13 & 0,04 & 75 & 89 & 97 \\
\hline 8 & $"$ & $"$ & 1 & 5 & 1 & 0,35 & 0,15 & 0,06 & 71 & 87 & 95 \\
\hline
\end{tabular}

\section{INFLUENCIA DA ALANTOINA NA PROTEÇÃO À OXIDAÇĀO}

Temperatura: $40^{\circ} \mathrm{C}$.

$$
\text { DO ÁCIDO ASCÓRBICO }
$$

Solução de sulfato de cobre: um $\mathrm{cm} .^{3}$ contendo um gama de ion cuprico.

Solução de Alatoina: com 100 gamas por $\mathrm{cm}^{3}$ da purina-derivado.

Solução aceto-acetica, tampão $0.2 \mathrm{~N}$ : $\mathrm{pH}$ 6, segundo Walpole.

Solução de ácido ascórbico: com poder redutor sobre o reativo de Tilmans F: 0.105 , de $19 \mathrm{~cm}^{3}$ para cada $\mathrm{cm}^{3}{ }^{3} 1.995 \mathrm{mgr}$. de ácido ascórbico por $\mathrm{cm} .{ }^{3}$

Volume total completado com agua, à $10 \mathrm{~cm} .^{3}$ em todos os tubos.

\begin{tabular}{|c|c|c|c|c|c|c|c|c|c|}
\hline \multirow[t]{2}{*}{ N.os } & \multicolumn{5}{|c|}{ Cm.3 da soluẹâo de } & \multicolumn{2}{|c|}{$\begin{array}{l}\text { Quantidade em } \\
\text { mgrs. de acido as- } \\
\text { córbico existente } \mathrm{em} \\
10 \mathrm{~cm} .3 \text { depoi. de }\end{array}$} & \multicolumn{2}{|c|}{$\begin{array}{l}\text { Perdas em por- } \\
\text { centagens de- } \\
\text { pois de }\end{array}$} \\
\hline & $\begin{array}{l}\text { Ácido } \\
\text { ascór. }\end{array}$ & Tampāo & $\begin{array}{l}\text { Sulfato } \\
\text { de cobre }\end{array}$ & $\begin{array}{l}\text { Alan- } \\
\text { toina }\end{array}$ & $\begin{array}{l}\text { Ascor- } \\
\text { binase }\end{array}$ & $15^{\circ}$ & $30^{\circ}$ & $15^{\circ}$ & $30^{\circ}$ \\
\hline 1 & 1 & 1 & - & - & - & 1,47 & 1,34 & 26 & 33 \\
\hline 2 & $"$ & " & 1 & - & - & 1,20 & 1,08 & 40 & 46 \\
\hline 3 & " & " & - & 5 & - & 1,62 & 1,38 & 19 & 31 \\
\hline 4 & ” & $"$ & 1 & 5 & - & 1,33 & 1,15 & 33 & 42 \\
\hline 5 & $"$ & $"$ & - & - & 1 & 1,01 & 0,87 & 49 & 53 \\
\hline 6 & $"$ & " & 1 & - & 1 & 1,01 & 0,87 & 49 & 53 \\
\hline 7 & $"$ & $"$ & - & 5 & 1 & 1,07 & 0,89 & 46 & 55 \\
\hline 8 & $"$ & $"$ & 1 & 5 & 1 & 0,53 & 0,48 & 73 & 76 \\
\hline
\end{tabular}


Vê-se assim que, nas condições do nosso trabalho, de todas as substâncias experimentadas apenas a xantina mostrou um resultado perfeitamente satisfatório; e sabendo-se que essa purina se encontra em diferentes tecidos animais fizemos uma prova para indagar do efeito protetor de um extrato de orgão, usando para isso figado. Valemo-nos de um extrato total do comercio equivalendo a uma concentração de $1: 10$ do orgão fresco.

\section{INFLUENCIA DO EXTRATO DE FIGADO NA PROTEÇÃO À OXIDAC̣ÃO \\ DO ÁCIDO ASCÓRBICO}

Temperatura: $40^{\circ} \mathrm{C}$.

Solução de sulfato de cobre: um $\mathrm{cm}^{3}$ contendo um gama de ion cuprico.

Soluções de extrato de figado: vêr nota abaixo do quadro.

Solução aceto-acetica, tampão $0.2 \mathrm{~N}: \mathrm{pH}$ 6, segundo Walpole.

Solução de ácido ascórbico: um $\mathrm{cm}^{3}$ reduziu $16.5 \mathrm{~cm} \cdot{ }^{3}$ do reativo de Tilmans, F. 0.097. 1,6 mgr. do ácido ascórbico para cada $\mathrm{cm} \cdot{ }^{3}$

Volume total completado com agua, à $10 \mathrm{~cm} \cdot{ }^{3} \mathrm{em}$ todos os tubos.

\begin{tabular}{|c|c|c|c|c|c|c|c|c|c|c|}
\hline \multirow[t]{2}{*}{ N.os } & \multicolumn{4}{|c|}{$\mathrm{Cm} .3$ da soluẹano de } & \multicolumn{3}{|c|}{$\begin{array}{l}\text { Qunntidade em mgrs. } \\
\text { de ácido aseórbico exis- } \\
\text { tente em } 10 \mathrm{em} .3 \\
\text { depois de }\end{array}$} & \multicolumn{3}{|c|}{$\begin{array}{l}\text { Perdas em porcenta- } \\
\text { gens depois de }\end{array}$} \\
\hline & $\begin{array}{l}\text { Ácido } \\
\text { ascór. }\end{array}$ & Tampão & $\begin{array}{l}\text { Sulfato } \\
\text { de cobre }\end{array}$ & $\begin{array}{l}\text { Mgr. de } \\
\text { figado }\end{array}$ & 15 & $30^{\circ}$ & $45^{\prime}$ & 15, & $30^{\circ}$ & $45^{\circ}$ \\
\hline 1 & 1 & 1 & - & - & 1,30 & 1,09 & 0,89 & 49 & 32 & 44 \\
\hline 2 & , & , & 1 & - & 0,88 & 0,60 & 0,31 & 45 & 62 & 80 \\
\hline 3 & $"$ & " & - & 500 & 1,60 & 1,60 & 1,60 & 0 & 0 & 0 \\
\hline 4 & $"$ & $"$ & 1 & 500 & 1,60 & 1,60 & 1,60 & 0 & 0 & 0 \\
\hline 5 & $"$ & $"$ & - & 100 & 1,60 & 1,60 & 1,60 & 0 & 0 & 0 \\
\hline 6 & , & ” & 1 & 100 & 1,60 & 1,60 & 1,60 & 0 & 0 & 0 \\
\hline 7 & " & $"$ & - & 50 & 1,60 & 1,60 & 1,60 & 0 & 0 & 0 \\
\hline 8 & " & $"$ & 1 & 50 & 1,60 & 1,55 & 1,55 & 0 & 1 & 3 \\
\hline 9 & ", & " & - & 33 & 1,60 & 1,60 & 1,60 & 0 & 0 & 0 \\
\hline 10 & $"$ & $"$ & 1 & 33 & 1,58 & 1,54 & 1,50 & 1 & 4 & 7 \\
\hline 11 & $"$ & $"$ & - & 6,5 & 1,60 & 1,60 & 1,60 & 0 & 0 & 0 \\
\hline 12 & " & " & 1 & 6,5 & 1,47 & 1,34 & 1,23 & 8 & 16 & 23 \\
\hline
\end{tabular}

NOTA: - Nos tubos que receberam figado, foram colocados $5 \mathrm{~cm} .3$ de diluiçáo de extrato de figado correspondente a $10 \mathrm{gr}$. de figado em $100 \mathrm{~cm} .3$ ou $0.1 \mathrm{gr}$. de figado para cada cm.3 (tubos 3 e 4); diluição correspondente a $0,02 \mathrm{gr}$. de figado $\mathrm{em} 1 \mathrm{~cm} .3 \mathrm{da}$ diluição (tubos 5 e 6 ): diluiçẩo correspondente a $0,01 \mathrm{gr}$. de figado $\mathrm{em} 1 \mathrm{~cm} .3 \mathrm{da}$ diluiçâo (tubos 7 e 8 ): diluição correspondente a $0.0066 \mathrm{gr}$. de figado em um $\mathrm{cm} .3$ da diluị̧āo (tubos 9 e 10): diluiçâo correspondente a $0.0033 \mathrm{gr}$. de figado $\mathrm{em}$ um $\mathrm{cm} .3$ da diluição (tubos 11 e 12 ). 
Verifica-se o elevado gráu de proteção do extrato de fígado contra a oxidação do ácido ascórbico, seja da expontanea seja da produzida pela ion cuprico. Ainda no equivalente a um peso de orgão fresco total a $16.5 \mathrm{mgr}$. no volume de $10 \mathrm{~cm} .^{3}$ as perdas foram apenas 18, 16, 23 por cento em presença de um gama de cobre, havendo proteção integral da oxidação expontanea.

Sabe-se que foi afirmada a existência de princípios antitoxicos do figado e que nas técnicas descritas por Forbes e colaboradores ficou evidenciado (9) que a xantina era a substância ativa na prevenção de animais pelo envenenamento pelo tetraclorêto de carbono. Pareceu-nos por isso, de conveniência, experimentar a atividade de um preparado obtido segundo a técnica descrita por ForBes em comparação com produtos farmaceuticos de existência no nosso arsenal terapeutico e que são obtidos segundo técnicas semelhantes.

Tais são os resultados:

\section{INFLUENCIA DOS ANTITOXICOS NA PROTEÇÃO À OXIDAÇÃO DO ÁCIDO ASCÓRBICO}

Temperatura: $40^{\circ} \mathrm{C}$.

Solução de sulfato de cobre: um $\mathrm{cm} \cdot{ }^{3}$ contendo um gama de ion cuprico.

Soluções de antitoxico (vêr nota do quadro anterior).

Solução aceto-acetica, tampão $0.2 \mathrm{~N}$ : pH 6, segundo Walpole.

Solução de ácido ascórbico: um $\mathrm{cm} .^{3}$ reduziu $16.5 \mathrm{~cm} .^{3}$ do reativo de Tilmans F. 0,097. $1.6 \mathrm{mgr}$. do ácido para cada $\mathrm{cm} \cdot{ }^{3}$

Volume total completado com agua, à $10 \mathrm{~cm} .^{3} \mathrm{em}$ todos os tubos.

\begin{tabular}{|c|c|c|c|c|c|c|c|c|c|c|}
\hline \multirow[t]{2}{*}{ N.oss } & \multicolumn{3}{|c|}{ Cm.3 da solução de } & \multirow{2}{*}{\begin{tabular}{|c|} 
Antito- \\
xico \\
corres- \\
ponden- \\
do a \\
mgr. de \\
figado
\end{tabular}} & \multicolumn{3}{|c|}{$\begin{array}{l}\text { Quantidade em mgr. } \\
\text { de ácido aseórbico } \\
\text { existente em } 10 \mathrm{~cm} .3 \\
\text { depois de }\end{array}$} & \multicolumn{3}{|c|}{$\begin{array}{l}\text { Perdas em porcenta- } \\
\text { gens depois de }\end{array}$} \\
\hline & $\begin{array}{l}\text { Acido } \\
\text { ascór. }\end{array}$ & Tampāo & $\begin{array}{l}\text { Sulfato } \\
\text { de cobre }\end{array}$ & & $15^{\circ}$ & $30^{\circ}$ & 45 & $15^{\circ}$ & $30^{\circ}$ & 45 \\
\hline 1 & 1 & 1 & - & - & 1,14 & 0,97 & 0,85 & 29 & 39 & 47 \\
\hline 2 & $"$ & $"$ & 1 & - & 0,83 & 0,72 & 0,63 & 48 & 55 & 61 \\
\hline 3 & ” & " & - & 50 & 1,26 & 1,06 & 0,98 & 29 & 33 & 39 \\
\hline 4 & " & " & 1 & 50 & 0,99 & 0,84 & 0,72 & 38 & 47 & 55 \\
\hline 5 & , & " & - & 33 & 1,25 & 1,15 & 0,97 & 22 & 28 & 39 \\
\hline 6 & " & 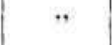 & 1 & 33 & 0,87 & 0,70 & 0,60 & 46 & 56 & 73 \\
\hline 7 & ” & $"$ & - & 16,5 & 1,16 & 0,93 & 0,80 & 27 & 42 & 50 \\
\hline 8 & ” & , & 1 & 16,5 & 0,81 & 0,73 & 0,65 & 49 & 54 & 59 \\
\hline 9 & " & , & - & 50 & 1,16 & 0,94 & 0,80 & 27 & 41 & 50 \\
\hline 10 & " & " & 1 & 50 & 0,90 & 0,76 & 0,63 & 44 & 52 & 61 \\
\hline 11 & ” & " & - & 33 & 1,16 & 0,92 & 0,81 & 27 & 42 & 50 \\
\hline 12 & " & ", & 1 & 33 & 0,86 & 0,75 & 0,65 & 46 & 53 & 59 \\
\hline 13 & " & " & - & 16,5 & 1,10 & 0,91 & 0,80 & 31 & 43 & 50 \\
\hline 14 & " & " & 1 & 16,5 & 0,68 & 0,56 & 0,41 & 57 & 65 & 74 \\
\hline
\end{tabular}


Pode-se comprovar por esses resultados que realmente, nos tres casos houve proteção contra a oxidação do ácido ascórbico, pelas chamadas frações anti-toxicas e preparadas no laboratório segundo tecnica de diferentes autores. Visando indagar agora sôbre o comportamento da xantina em face de outros agentes oxidantes realisamos a experimentação que segue:

\section{INFLUENCIA DA XANTINA NA PROTEÇÃO A OXIDAÇÃO DO ÁCIDO ASCÓRBICO PELOS IONS OXIDANTES CUPRICO E FÉRRICO E PELO PEROXIDO DE HIDROGENIO}

Temperatura: $40^{\circ} \mathrm{C}$.

Solução de sulfato de cobre: um $\mathrm{cm}^{3}$ contendo um gama de ion cuprico.

Solução de xantina: contendo por $\mathrm{cm} \cdot{ }^{3} 70$ gamas da dioxipurina.

Solução aceto-acetica, tampão $0.2 \mathrm{~N}$ : pH 6, segundo Walpole.

Solução de ácido ascórbico: um cm. ${ }^{3}$ reduziu $18,0 \mathrm{~cm} \cdot{ }^{3}$ do reativo de Tilmans F. 0.097: 1,833 mgi. de ácido ascórbico por $\mathrm{cm} \cdot{ }^{3}$

Solução férrica: com 5 gamas por $\mathrm{cm}^{3}$ de ion férrico.

Solução de peroxido de hidrogenio: capaz de fornecer 14 gamas de oxigenio atomico por $\mathrm{cm} .^{3}$

Volume total completado com agua, à $10 \mathrm{~cm} \cdot{ }^{3} \mathrm{em}$ todos os tubos.

\begin{tabular}{|c|c|c|c|c|c|c|c|c|c|c|c|c|}
\hline \multirow[b]{2}{*}{ N.os } & \multicolumn{6}{|c|}{ Cm.3 da solução de } & \multicolumn{3}{|c|}{$\begin{array}{l}\text { Quantidade em mgr. } \\
\text { de ácido ascórbico } \\
\text { existente depois de }\end{array}$} & \multicolumn{3}{|c|}{$\begin{array}{l}\text { Perdas em porcenta- } \\
\text { gens depois de }\end{array}$} \\
\hline & $\begin{array}{l}\text { Ácido } \\
\text { ascór. }\end{array}$ & $\begin{array}{c}\text { Tam- } \\
\text { pão }\end{array}$ & Cobre & Ferro & $\begin{array}{l}\text { Pero- } \\
\text { xido } \\
\text { de } \\
\text { hidro- } \\
\text { genio }\end{array}$ & $\begin{array}{l}\text { Xan- } \\
\text { tins }\end{array}$ & $15^{\prime}$ & $30^{\prime}$ & $45^{\prime}$ & $15^{\prime}$ & $30^{\circ}$ & $45^{\prime}$ \\
\hline 1 & 1 & 1 & - & - & - & - & 1,42 & 1,33 & 1,21 & 22 & 27 & 34 \\
\hline 2 & " & $"$ & 1 & - & - & - & 1,05 & 0,95 & 0,76 & 42 & 48 & 58 \\
\hline 3 & " & " & 1 & - & - & 5 & 1,54 & 1,37 & 1,22 & 16 & 25 & 33 \\
\hline 4 & , & " & - & 1 & - & - & 1,04 & 0,80 & 0,78 & 43 & 56 & 57 \\
\hline 5 & $"$ & ", & - & 1 & - & 5 & 1,62 & 1,55 & 1,52 & 11 & 15 & 17 \\
\hline 6 & " & $"$ & - & - & 1 & - & 1,06 & 0,85 & 0,67 & 42 & 54 & 63 \\
\hline 7 & " & " & - & - & 1 & 5 & 1,59 & 1,52 & 1,48 & 13 & 17 & 19 \\
\hline
\end{tabular}

Pode-se bem observar a perfeita eficiência da xantina na proteção do ácido ascórbico, ainda ligeiramente aumentada se o agente oxidante é o férro ou o peroxido de hidrogenio.

\section{DISCUSSÃO}

A grande diferença observada entre a ação protetora da xantina na oxidação do ácido ascórbico e outros compostos correlatos, indica tratar-se de um efeito especifico, e portanto independente de um determinado grupamento funcional. Não parece razoável admi- 
tir, como fazem KrishnamurThy e GIRI (1) que ação protetora dependa do imino-grupo livre em posição 7; inclusive, nas condições de nosso trabalho o ácido úrico não apresentou eficiencia apreciavel. Escolhemos preferentemente $o \mathrm{pH}=6$ para o nosso trabalho por ser essa condição habitual nas dosagens do ácido ascórbico com o emprego da ascorbinase. Ficou evidenciado que nenhuma das substâncias experimentadas poderá ser responsabilisada pela inhibição da ascorbinase em dosagens de vitamina $\mathrm{C}$, segundo fôra observado por Pimenta (5). Os resultados obtidos com o extrato de figado sugerem a coexistência de outra ou outras substâncias protetoras do ácido ascórbico que não a xantina apenas, já que quantidades extraordinariamente pequenas do orgão mostram um efeito maior que o que seria de se admitir quando considerada sua porcentagem em xantina; seria talvez admissivel que no extrato de figado a xantina se apresentasse em forma combinada e que nestas circunstancias seu efeito fosse mais intenso que na forma livre. Tambem deve ser considerado que o efeito protetor da xantina e dos extratos purificados de figado de acordo com as técnicas do tipo de ForBES poderiam mostrar "in vivo" uma ação semelhante que talvez servisse como indicação para explicar o mecanismo de ação da chamada fração anti-toxica do figado. Lembremo-nos a proposito que o ácido ascórbico age como desintoxicante do organismo com respeito a substancias as mais variadas, sejam compostos inorganicos de mercurio ou de arsenico, sejam venenos organicos ou organo-metalicos (1-tirosina, arseno-benzois, etc.) ou mesmo substancias biologicas como toxinas diftericas e tetanicas. Nada se sabe de positivo sobre a maneira de agir o ácido ascórbico como desentoxicante e não é sem fundamento que se pode admitir que o ácido ascórbico sendo protegido "in vivo" pela xantina ou correlatos, seria mais eficiente no seu papel desentoxicante; com isso talvês a atuação da fração anti-toxica do figado fosse de um tipo indireto, agindo por intermedio do ácido ascórbico.

\section{RESUMO}

Confirma-se a eficiência da xantina na proteção do ácido ascórbico contra certos agentes oxidantes tais como os ions cuprico, férrico e o peroxido de hidrogenio. Este efeito protetor, trabalhando em $\mathrm{pH}$ 6 e a $40^{\circ} \mathrm{C}$., é falho em outras substâncias examinadas como o ácido úrico, guanina, creatinina, veronal e alantoina.

Não existe manifestação do efeito da xantina na oxidação do ácido ascórbico pela ascorbinase; esta, praticamente não é alterada na sua capacidade de ação pela presença da xantina embora seja evidente 
de que o ion cuprico, por si só, diminúe a potencia da ascorbinase. Os extratos de figado, totais ou purificados segundo tecnica de preparação das chamadas frações anti-toxicas, são extraordinariamente ativos na proteção do ácido ascórbico e esse fato sugere para tais substancias um papel indireto de desentoxicação no organismo o que se cumpriria por intermedio do ácido ascórbico.

\section{SUMMARY}

The efficiency of xanthine on the protection of ascorbic acid against certain oxidating agents, such as cupric and ferric ions and hydrogen peroxide, is confirmed. This protecting effect, working at $p H 6$ and at $40^{\circ} \mathrm{C}$ is failable with other substances examined, such as uric acid, guanine, creatinine, veronal and alantoine.

There is no manifestation of the effect of xanthine on the oxidation of ascorbic acid by ascorbinase; this is not practically altered in its capacity of action by the presence of xanthine, though it is evident that the cupric ion itself decreases the power of the ascorbinase. Liver extracts, total or purified according to the technique of preparation of the so called anti-toxic fractions, are extraordinarily active in the protection of ascorbic acid and this fact suggests, por such substances, an indirect desintoxicating role in the organism, which would be accomplished by means ascorbic acid.

\section{BIBLIOGRAFIA}

1) Giri e Krishnamurthy - $1941-$ Nature 147: 59.

2) Musulin, R. R. - King, G. G. - 1936 - Jour. Biol. Chem. 116: 409.

3) Lyman C. M., Shultz M. O. - King, G. G. - 1937 - Jour. Biol. Chem. 118: 757 .

4) Riberro, O. F. - 1941 - Rev. Fac. Med. Vet. de S. Paulo 2, (1): 3-7.

5) Prmenta, N. - 1941 - Tése para catedratico à Faculdade de Farmacia e Odontologia de São Paulo.

6) Ribeiro, R. F. - Bonoldi, V. - Ribeiro, O. F. - 1942 - Rev. Fac. Med. Vet. de S. Paulo 2, (2) : 29-39.

7) Leser, W. P. - 1941 - Tése para catedratico à Escola Paulista de Medicina.

8) Tauber, H. - Kleiner, I. - 1935 - Jour. Biol. Chem. 110: 559.

9) Forbes, J. C. e Jeanette S. Mc. Connell - 1937 - Proc. Soc. Exptl. Biol. Med. 36: 359-60. 